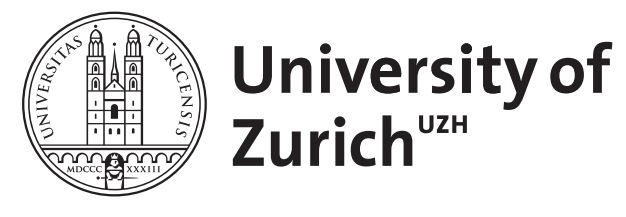

\title{
Schlafstörungen bei Demenzkranken
}

\author{
Savaskan, E
}

\begin{abstract}
Dementia is characterized by cognitive and also behavioral and psychological symptoms of dementia (BPSD). The most prominent BPSD are depression and apathy but sleep disorders also complicate the clinical course of dementia. These symptoms are a severe burden for patients and caregivers and are difficult to treat partly due to comorbidities. Common sleep disorders in dementia are insomnia, hypersomnia, circadian rhythm alterations and aberrant nocturnal motor behavior. Sleep duration and rapid eye movement (REM) sleep are reduced. The diagnostic assessment of sleep disorders should include an evaluation of the underlying risk factors and a detailed sleep history for which several assessment instruments are available. The therapy of sleep disorders of dementia is primarily nonpharmacological: sleep counseling, sleep hygiene regulation, relaxation and psychotherapy techniques are given priority. Pharmacological treatment often has severe side effects in this elderly, vulnerable population and can only be considered if other nonpharmacological options have been unsuccessful. The application of medication should be limited in time and dosage. The pharmacological therapeutic options are critically discussed in detail.
\end{abstract}

DOI: https://doi.org/10.1007/s00391-015-0890-3

Other titles: Sleep disorders in dementia patients

Posted at the Zurich Open Repository and Archive, University of Zurich ZORA URL: https://doi.org/10.5167/uzh-117739

Journal Article

Published Version

Originally published at:

Savaskan, E (2015). Schlafstörungen bei Demenzkranken. Zeitschrift für Gerontologie und Geriatrie, $48(4): 312-317$.

DOI: https://doi.org/10.1007/s00391-015-0890-3 
Z Gerontol Geriat 2015 · 48:312-317

DOI 10.1007/s00391-015-0890-3

Eingegangen: 29. September 2014

Überarbeitet: 18. Dezember 2014

Angenommen: 1. April 2015

Online publiziert: 9. Mai 2015

(c) Springer-Verlag Berlin Heidelberg 2015

\section{E. Savaskan}

Klinik für Alterspsychiatrie, Psychiatrische Universitätsklinik Zürich, Zürich, Schweiz

\section{Schlafstörungen bei Demenzkranken}

\section{Pathophysiologie}

havioralen und psychologischen Symptomen der Demenz (BPSD). Diagnose und Therapie von Schlafstörungen bei an Demenz Erkrankten bedeuten eine besondere Herausforderung, da die oft bestehenden Komorbiditäten und das Alter der Patienten neben der eigentlichen Erkrankung erschwerende Faktoren darstellen.

Außer kognitiven Störungen weisen Patienten mit Demenzerkrankungen, wie Alzheimer-Demenz (AD) als prominenteste Form, eine Reihe von behavioralen und psychologischen Symptomen der Demenz (BPSD) auf. Apathie und Depression sind die häufigsten BPSD.

Schlafstörungen sind mit einer Prävalenz von $26-50 \%$ ein wichtiges BPSD.

Sie erschweren besonders den klinischen Verlauf $[7,21]$. Nicht nur AD, sondern auch andere neurodegenerative Erkrankungen wie Lewy-Körperchen-Demenz, frontotemporale Demenz sowie Demenz bei M. Parkinson und sogar seltenere Demenzformen können ausgeprägte Schlafstörungen verursachen [21]. Die Schlafstörung kann tagsüber zu Müdigkeit, Somnolenz, Irritabilität, Kopfschmerzen, Depression sowie zur zusätzlichen Verschlechterung der motorischen und der kognitiven Fähigkeiten führen. Die ohnehin eingeschränkten Alltagsfähigkeiten der Patienten werden weiter beeinträchtigt [5]. Auch die Betreuer sind betroffen und leiden vermehrt unter Stress und Depression $[5,15]$.
Schlafstörungen bei Demenz, insbesondere bei $\mathrm{AD}$, können als Insomnie, Hypersomnie, Schlafapnoe, exzessive nächtliche motorische Aktivität, zirkadiane Schlaf-Wach-Rhythmusstörungen und als „sun downing“, mit am Abend erscheinenden Unruhezuständen, auftreten [5]. Die Fragmentierung des nächtlichen Schlafs mit vermehrten nächtlichen Wachphasen, reduzierter REM-Schlafphase, Abnahme der nächtlichen Schlafdauer, Auftreten von Kurzschlafphasen und Somnolenz während des Tages sind typisch für AD [19]. Während die anderen Schlafstörungen auch bei gesunden älteren Personen vorkommen können, sind Störungen der REM-Schlafphase spezifisch für Demenz. Die frühe REMSchlaf-Störung wird auch als ein Indikator für später auftretende neurodegenerative Erkrankungen, v. a. aus der Gruppe der $\alpha$-Synukleopathien, erachtet.

\section{》) Störungen der \\ REM-Schlafphase sind spezifisch für Demenz}

Die bestehende Interaktion zwischen dem Schlafapnoesyndrom und Demenz ist sehr komplex, weil dieses Syndrom als unabhängiger Risikofaktor für Demenz gilt und zu kognitiven Verschlechterungen beim Patienten führen kann [19]. Die Prävalenz des Schlafapnoesyndroms nimmt im Alter und insbesondere bei Vorliegen einer $\mathrm{AD}$ zu und kann bei AD-Patienten $40-70 \%$ betragen. Die Schlafstörungen bei Lewy-Körperchen-Demenz ähneln denen bei $\mathrm{AD}$, aber Insomnie, Hypersomnolenz tagsüber und REM-Schlafstörungen überwiegen. Diese Patienten zeigen nächtliche visuelle Halluzinationen.
Im Rahmen einer Demenz bei M. Parkinson stehen Schläfrigkeit, Müdigkeit und Somnolenz am Tag mit gelegentlichen Schlafattacken im Vordergrund. Insomnie, respiratorische Störungen während des Schlafs und Restless-legs-Syndrom (RLS) in der Nacht sind sehr häufig. Die häufigsten Schlafbeschwerden bei Patienten mit M. Parkinson sind nächtliches Aufwachen und Schlaffragmentation [22]. Störungen der REM-Schlafphase treten bei diesen Patienten sehr häufig auf; dies sind oft mit exzessiver nächtlicher motorischer Aktivität verbunden.

Die neurobiologische Grundlage der Schlafstörungen bei Demenz bilden die strukturellen Veränderungen in Hypothalamus und Hirnstamm sowie Degenerationsprozesse in den cholinergen Neuronen des Nucleus basalis (Meynert-Kern) und im suprachiasmatischen hypothalamischen Nucleus [5]. Indirekte Faktoren wie komorbide Erkrankungen, respiratorische Dysrhythmie, Medikamenteneinnahme, Immobilität und Nykturie können vorhandene Schlafstörungen verstärken. Bei AD und bei Demenz im Rahmen des M. Parkinson ist der Zusammenhang zwischen Amyloid- $\beta$-Peptid- $\left(\mathrm{A}_{\beta}\right)$ - und $\alpha$-Synuklein-Ablagerungen in Hirnregionen, die in die Schlafregulation involviert sind, und der Schlafstörung gut belegt [22]. $A_{\beta}$-induzierte neuropathologische Prozesse verstärken die Schlafstörungen. Es sind auch Hinweise vorhanden, dass Schlafstörungen die Konzentration von löslichem $A_{\beta}$ erhöhen und dadurch die Neurodegeneration vorantreiben können [14]. Veränderungen in Neurotransmitter- und Hormonsystemen wie z. B. Serotonin, Norepinephrin und Melatonin sowie beim Nervenwachstumsfaktor „Brain-derived neurotrophic factor" (BDNF) tragen ebenfalls zur Schlafstörung bei. 


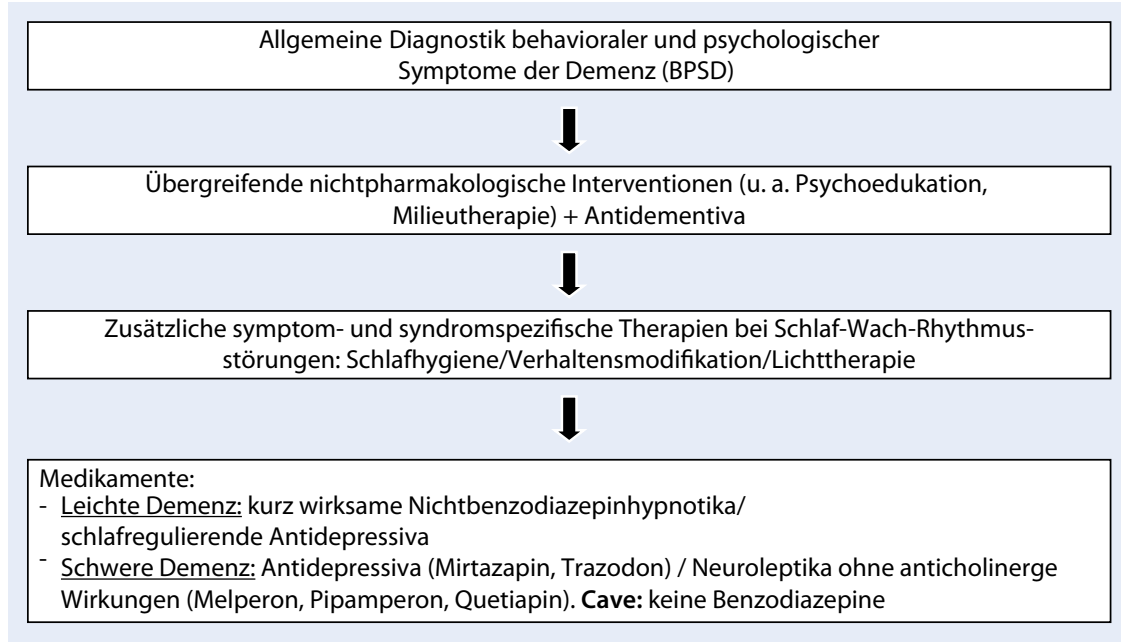

Abb. $1 \Delta$ Evidenzbasierte Empfehlungen zur Behandlung von Störungen des Schlaf-Wach-Rhythmus bei Demenzkranken. (Modifiziert nach [24])

\section{Diagnose}

Der erste Schritt der Diagnostik ist die ausführliche Schlafanamnese mit Erhebung der Vorgeschichte und aktueller Probleme. Hierbei wird auf Symptome und zugrunde liegende neurodegenerative Erkrankungen fokussiert [5, 21]. Neuropsychiatrische Symptomatik, Familienanamnese, Medikation und Abhängigkeitserkrankungen müssen eruiert werden. Ein Leitfaden zur individualisierten Erfassung von Schlafgewohnheiten und Risikofaktoren bei Demenzpatienten kann der Publikation von Roth [21] entnommen werden und erweist sich im klinischen Alltag als sehr nützlich [21].

Der Anamnese folgt die detaillierte klinische und neurologische Untersuchung mit dem Ziel, mögliche Komorbiditäten als Ursache der Schlafstörung auszuschließen. Eine Insomnie kann eine Reihe internistischer, neurologischer, physiologischer und pharmakologischer Ursachen haben, die abgeklärt und ggf. behandelt werden müssen [12]. Zum Beispiel können kardiovaskuläre und pulmonale Erkrankungen, gynäkologische Grunderkrankungen oder die Einnahme von Medikamenten (aktivierende Antidepressiva, Antiasthmatika, Antibiotika, Anticholinergika, Antihypertensiva, Migräne- oder Anti-Morbus-Parkinson-Mittel) und Substanzen wie Alkohol oder Kaffee Schlafstörungen verursachen. Falls die Abklärung der Demenzerkrankung nicht vollständig ist, muss dies nachgeholt wer- den. Differenzialdiagnostisch müssen ein Schlafapnoesyndrom und die Pseudodemenz bei Depression ausgeschlossen werden.

\section{》) Zirkadiane Schlaf-Wach- Rhythmusstörungen mithilfe der Aktographie dokumentieren}

Weiterführende Diagnostikinstrumente für die Schlafstörung stellen die Polysomnographie und der Multiple Wachbleibetest dar [5, 12]. Für die Anamnese werden der „Pittsburgh Schlafqualitätsindex“ (PSQI; erhältlich unter: http://www.charite.de) und die „Epworth Sleepiness Scale“ sowie die Führung eines Schlaftagebuchs empfohlen. Bei Demenzpatienten, die schwierige Untersuchungen nicht zulassen, kann eine Aktographie hilfreich sein. Mithilfe der Aktographie können zirkadiane Schlaf-Wach-Rhythmusstörungen gut dokumentiert werden.

\section{Therapie}

In der Therapie der Schlafstörungen bei Demenz muss zwischen ursachen- und symptomorientierten sowie zwischen nichtpharmakologischen und pharmakologischen Interventionen unterschieden werden [5, 12]. Einige Faktoren, die Schlafqualität beeinträchtigen, wie z. B. medizinische oder psychiatrische Komorbiditäten, Lebensstil und Schlafhygiene, müssen vor Therapiebeginn eruiert wer- den. Eine ursachenorientierte Abklärung sollte auf jedem Fall einer Therapie vorangehen, um die Ursache der Insomnie spezifisch zu behandeln [12]. Die symptomatische Therapie der Schlafstörungen kann dann zusätzlich angeboten werden.

\section{ע) Nichtpharmakologi- sche Interventionen vor der medikamentösen Behandlung ansetzen}

Die pharmakologischen Behandlungsmöglichkeiten, die im Folgenden behandelt werden, richten sich an den Therapieempfehlungen der Schweizer Fachgesellschaften für BPSD, die Anfang 2014 publiziert wurden ( $\bullet$ Abb. 1; [24]), aus. Die Therapieempfehlungen der Schweizer Fachgesellschaften basieren auf der S3-Leitlinie „Demenzen“ der Deutschen Gesellschaft für Psychiatrie, Psychotherapie und Nervenheilkunde (DGPPN) und der Deutschen Gesellschaft für Neurologie (DGN; [25]). Nichtpharmakologische Interventionen müssen gemäß den Empfehlungen vor der medikamentösen Behandlung ansetzen und diese zumindest begleiten.

\section{Nichtpharmakologische Verfahren}

Die Regeln der Insomniebehandlung gelten für die Therapie aller Formen von Schlafstörungen. Die Insomniebehandlung basiert auf Aufklärung und Beratung der Betroffenen sowie deren Betreuer.

\section{\) Schlafhygieneregeln vermitteln}

Wichtiges Element ist die Vermittlung von Schlafhygieneregeln [12]. Zusammengefasst beinhalten diese:

- körperliche Tätigkeit tagsüber ohne Überforderung ausführen,

- leichte Mahlzeiten zu sich nehmen,

- warm und kalt duschen,

- Kaffee, Alkohol und andere Stimulanzien vermeiden,

- dunkle und ruhige Atmosphäre im Schlafzimmer schaffen,

- zu regelmäßigen Zeiten zu Bett gehen und aufstehen, 
- kein Mittagsschlaf und nur knapp bemessene Schlafdauer,

- statt sich schlaflos im Bett zu wälzen, besser aufstehen,

- Paradoxie der Schlaferwartungshaltung durchbrechen,

- Schlafmittel möglichst vermeiden.

Entspannungsverfahren unterstützen die Insomnietherapie. Verhaltenstherapeutische Verfahren mit Stimuluskontrolle, Schlafrestriktion, paradoxer Intervention und kognitiven Techniken sowie Entspannungsverfahren wie progressive Muskelrelaxation, autogenes Training, Yoga und Meditation oder/und die interpersonelle Psychotherapie sind im frühen Demenzstadium gut wirksam [12].

Bei Patienten mit Demenz und Insomnie muss zusätzlich erwogen werden, die Einnahme von Medikamenten, die zur Schlafstörung beitragen oder diese verursachen, zu reduzieren oder ganz abzusetzen. Komorbide Depression, Angst und Schmerzen sind entsprechend zu behandeln, und morgens sowie abends erweist sich die Exposition hellen Lichts als wirksam. Regelmäßige körperliche Aktivität sowie ein ruhiges und aktivierendes Umfeld wirken sich positiv aus [5].

\section{Pharmakologische Therapie}

Ältere Menschen mit Demenz reagieren aufgrund ihres Allgemeinzustands, der Multimorbidität und der häufig damit verbundenen Polypharmazie sehr empfindlich auf Medikamente. Nebenwirkungen kommen in dieser Patientengruppe besonders zum Tragen.

$>$ Die Verabreichung von Psychopharmaka muss nach klarer Indikation, zeitlich begrenzt und unter klinischer Beobachtung erfolgen.

Folgende Grundsätze der Psychopharmakatherapie bei BPSD müssen eingehalten werden $[18,24]$ :

- In erster Linie sollen nichtpharmakologische Therapien eingesetzt werden.

- Ein individueller Therapieplan soll erstellt werden.

- Die bestehende Medikation sollte vor Beginn einer neuen Medikation be-

Z Gerontol Geriat 2015 · 48:312-317 DOI 10.1007/s00391-015-0890-3

○ Springer-Verlag Berlin Heidelberg 2015

\section{E. Savaskan}

\section{Schlafstörungen bei Demenzkranken}

\section{Zusammenfassung}

Demenzerkrankungen weisen neben kognitiven Symptomen eine Reihe von behavioralen und psychologischen Symptomen der Demenz (BPSD) auf. Depression und Apathie sind die häufigsten BPSD, aber auch Schlafstörungen, die den klinischen Verlauf erschweren, kommen vor. Diese Symptome stellen eine starke Belastung für Betroffene und deren Betreuer dar. Oft sind sie aufgrund der bestehenden Komorbiditäten schwierig zu behandeln. Häufige Formen der Schlafstörungen bei Demenz sind Insomnie, Hypersomnie, zirkadiane Rhythmusstörungen und nächtliche motorische Unruhe. Schlafdauer und "Rapid-eye-movement "(REM)-Schlafphase sind reduziert. Die Diagnostik der Schlafstörungen bei Demenz muss die Risikofaktoren abklären und eine detaillierte Schlafanamnese beinhalten; verschiedene Instrumen- te sind hierzu verfügbar. Die Therapie ist in erster Linie nichtpharmakologisch: Aufklärung und Beratung, Vermittlung von Maßnahmen der Schlafhygiene, verschiedene Entspannungstechniken und psychotherapeutische Interventionen stehen im Vordergrund. Pharmakologische Therapien gehen oft mit schweren Nebenwirkungen bei dieser älteren, vulnerablen Patientengruppe einher und dürfen nur eingesetzt werden, wenn nichtpharmakologische Optionen nicht ausreichen. Der Einsatz der Medikamente muss zeitlich und in der Dosierung limitiert erfolgen. Die pharmakologischen Therapiemöglichkeiten werden im Detail kritisch diskutiert.

\section{Schlüsselwörter}

Insomnie · Alzheimer-Krankheit · Schlaf, REM . Inadäquate Schlafhygiene - Therapeutik

\section{Sleep disorders in dementia patients}

\section{Abstract}

Dementia is characterized by cognitive and also behavioral and psychological symptoms of dementia (BPSD). The most prominent BPSD are depression and apathy but sleep disorders also complicate the clinical course of dementia. These symptoms are a severe burden for patients and caregivers and are difficult to treat partly due to comorbidities. Common sleep disorders in dementia are insomnia, hypersomnia, circadian rhythm alterations and aberrant nocturnal motor behavior. Sleep duration and rapid eye movement (REM) sleep are reduced. The diagnostic assessment of sleep disorders should include an evaluation of the underlying risk factors and a detailed sleep history for which several assessment instruments are available. The therapy of sleep disorders of dementia is primarily nonpharmacological: sleep counseling, sleep hygiene regulation, relaxation and psychotherapy techniques are given priority. Pharmacological treatment often has severe side effects in this elderly, vulnerable population and can only be considered if other nonpharmacological options have been unsuccessful. The application of medication should be limited in time and dosage. The pharmacological therapeutic options are critically discussed in detail.

\section{Keywords}

Insomnia · Alzheimer's disease · Sleep, REM . Inadequate sleep hygiene - Therapeutics züglich Nebenwirkungen und Interaktionen geprüft werden.

- Wenn möglich sollte eine Monotherapie erfolgen.

- Substanzen sollten nacheinander in das Therapieregime aufgenommen werden.

- Wenn möglich sollten Rezeptorantagonisten wie Anticholinergika und Antihistaminika sowie Dopaminagonisten vermieden werden.

- Tiefe Startdosis und langsames Aufdosieren sollten beachtet werden.
- Aufklärung über die Therapie und die Kontrolle der Medikamenteninteraktionen sollte integraler Bestandteil der Therapie sein.

Ein Grundproblem der medikamentösen Behandlung der Schlafstörungen bei Demenz ist, dass für die bestehenden Optionen kaum kontrollierte Studien existieren und die klinische Evidenz nicht ausreichend ist. Trotzdem ist für einige Substanzen eine gute therapeutische Erfahrung vorhanden, und diese können unter 


\section{Hier steht eine Anzeige.}

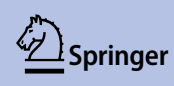

Berücksichtigung des Nebenwirkungspotenzials wirkungsvoll eingesetzt werden. Die Verabreichung vieler Substanzen erfolgt außerhalb der Indikation als „off label use“. Entsprechende Richtlinien müssen eingehalten werden. Im Folgenden werden die vorhandenen pharmakologischen Optionen kritisch diskutiert.

\section{Benzodiazepine}

Weiterhin gehört diese Substanzgruppe zu den am meisten verordneten Medikamenten bei älteren Menschen und Demenzpatienten. Benzodiazepine sind zwar wirksame Schlafmittel, gehen aber mit einer Reihe von schwerwiegenden Nebenwirkungen wie vermehrter Sturzneigung, Muskelrelaxation, Toleranzentwicklung, Atemdepression und Abhängigkeit einher $[10,24]$. Bei Demenz kann sich die kognitive Symptomatik unter Benzodiazepingaben verschlechtern und ein Delir auftreten. Neuere Studien bezweifeln die schlaffördernde Wirkung dieser Substanzen im Alter [3] und betonen, dass der Einsatz bei älteren Personen mehr schadet als Nutzen bringt $[1,10]$. Deswegen werden Benzodi- azepine grundsätzlich für diese Indikation nicht empfohlen. Falls diese Substanzen in Notfällen trotzdem eingesetzt werden müssen, sollen um einer Kumulationsgefahr vorzubeugen, nur kurz wirksame Benzodiazepine, wie Lorazepam, und zeitlich limitiert (bis maximal 3 Wochen) verabreicht werden.

\section{Benzodiazepinanaloga}

Benzodiazepinanaloga wie Zopiclon, Zolpidem und Zaleplon werden bei Schlafstörungen oft eingesetzt und sind ähnlich wirksam wie Benzodiazepine [10, 21, 23, 24]. Die Nebenwirkungen der Benzodiazepine sind bei diesen Substanzen weniger ausgeprägt, aber Stürze der Patienten kommen häufig vor. Nebenwirkungen überwiegen den Nutzen, und der zeitlich limitierte Einsatz ist nur gerechtfertigt, wenn alle anderen Maßnahmen nicht zum Ziel geführt haben.

\section{Antidepressiva}

Hypnotisch wirksame Antidepressiva wie Mirtazapin und Trazodon haben positive Wirkungen auf die Schlafdauer und die nächtliche Wachzeit [4, 20, 21, 23, 24]. Eine aktuelle Cochrane-Analyse der Studien über die Pharmakotherapie der Schlafstörungen bei $\mathrm{AD}$ kam zu dem Schluss, dass nur Trazodon die nächtliche Schlafzeit signifikant verlängert [14]. Die Präparate werden deswegen oft als schlaffördernde Substanzen eingesetzt. Wenn der Grund der Schlafstörung eine Depression ist, sollte diese grundsätzlich behandelt werden. Bei Demenzpatienten dürfen Antidepressiva mit anticholinergem Nebenwirkungspotenzial nicht eingesetzt werden.

\section{》) Selektive Serotonin-Wieder- aufnahmehemmer bevorzugen}

Antidepressive Substanzen wie selektive Serotonin-Wiederaufnahmehemmer („selective serotonin reuptake inhibitor", SSRI) sind aufgrund ihrer günstigeren Nebenwirkungsprofile zu bevorzugen. Auch die hypnotisch wirksamen Antidepressiva können bei Demenzpatienten Nebenwirkungen wie Somnolenz, Sedation und Schwindel verursachen und müs- 
sen klinisch überwacht werden. Mirtazapin kann ein RLS verstärken.

\section{Melatonin und Melatoninagonisten}

Obwohl die Datenlage noch inkonsistent ist, ist die Wirksamkeit des Melatonins bei zirkadian bedingten demenziellen Schlafstörungen insgesamt belegt $[6,9,24]$. Vor allem in Kombination mit einer Lichttherapie scheint es gut zu wirken. Melatonin ist bei über 55-Jährigen für die Behandlung von Schlafstörungen zugelassen. Eine aktuelle Cochrane-Analyse kam aber zu dem Ergebnis, dass aktuell keine Evidenz dafür besteht, dass Melatonin den Schlaf von AD-Patienten verbessert [14]. Nebenwirkungen sind Reizbarkeit, Nervosität, Albträume sowie psychomotorische Unruhe, und diese sind manchmal schwierig von Symptomen der Schlafstörung zu unterscheiden.

\section{Antipsychotika}

Niederpotente Antipsychotika wie Pipamperon und Melperon (Letzteres in der Schweiz nicht zugelassen) sowie atypische Antipsychotika wie Risperidon oder Quetiapin werden bei Patienten mit Demenz oft zur Behandlung von nächtlichen Unruhezuständen und Schlafstörungen eingesetzt $[2,23,24]$. Dabei wird die sedative Wirkung dieser Substanzen ausgenutzt. Diese Medikamente haben allerdings schwerwiegende Nebenwirkungen wie Sedation, vermehrte Sturzneigung, extrapyramidale Effekte oder anticholinerge unerwünschte Wirkungen in Form von Herz-Kreislauf-Problemen und Harnverhalt. Untersuchungen belegen, dass die Mortalität von Demenzpatienten und das Risiko für zerebrovaskuläre Ereignisse beim Einsatz dieser Substanzen erhöht sind. Deswegen sind sie lediglich bei guter klinischer Expertise und klarer Indikation nur zeitlich limitiert (bis 6 Wochen), in niedrigster Dosierung und, wenn Alternativen fehlgeschlagen sind, einzusetzen.

\section{Chloralhydrat}

Chloralhydrat wurde v. a. früher sehr oft als Schlafmittel eingesetzt, jedoch existieren keine kontrollierten Studien mit dieser Substanz [11]. Es hat eine geringe therapeutische Breite und zeigt einen raschen Wirkungsverlust. Für ältere und Demenz- patienten ist die Gabe von Chloralhydrat nicht zu empfehlen.

\section{Weitere Substanzen}

Medikamente wie Diphenhydramin, Promethazin, Doxylamin, Hydroxyzin, Chlorpheniramin und trizyklische Antidepressiva haben wegen starken anticholinergen Nebenwirkungen keinen Platz in der Behandlung der Schlafstörungen des alten und an Demenz erkrankten Patienten $[8,24]$. Chlomethiazol sollte wegen seiner atemdepressiven Wirkung nicht verabreicht werden.

\section{Antidementiva}

Antidementiva haben nicht nur kognitive Effekte sondern, zeigen auch gute Wirksamkeit bei BPSD. Vor allem für den Cholinesterasehemmer Donepezil existieren einige Studien über eine schlafregulierende Wirkung bei AD [16, 17].

> Vor allem die REM-Schlafphase wird verbessert.

Dies kann indirekt die Kognition stabilisieren. Die Schlafzeit insgesamt nimmt $\mathrm{zu}$, und eine Schlafapnoe kann verbessert werden. Es empfiehlt sich, das Medikament morgens zu applizieren, da abendliche Dosierungen nächtliche Unruhezustände und Schlafstörungen verursachen können. Es besteht noch wenig klinische Erfahrung mit Antidementiva bei Schlafstörungen.

\section{Phytotherapeutika}

Einige pflanzliche Substanzen, v. a. Baldrian, sind in ihrer Wirkung bei Schlafstörungen, nicht aber bei Demenz untersucht $[22,23,26]$. Die Ergebnisse sind insgesamt uneinheitlich, aber Phytotherapeutika zeichnen sich durch geringe Toxizität und wenige Nebenwirkungen aus. Bei Demenz besteht noch keine Empfehlung.

\section{Weitere Therapieverfahren}

Die Lichttherapie ist ein Verfahren, das kaum Nebenwirkungen aufweist. Sie kann gut in den Alltag von Demenzpatienten integriert werden. Dies gilt sowohl für die über eine definierte Zeit stattfindende Applikation unter Anwendung einer Thera- pielampe als auch für die Lichtexposition, die durch eine bessere Deckenbeleuchtung in Aufenthalts- und Therapieräumen erreicht werden kann.

) Lichttherapie kann gut in den Alltag von Demenzpatienten integriert werden

Letzteres ist v. a. für Patienten mit fortgeschrittener Demenz von Belang, die aufgrund der Schwere ihrer kognitiven Störung nicht lange genug vor einer Lampe sitzen bleiben. Auch wenn die klinische Evidenz noch nicht ausreichend ist, zeigen Untersuchungen, dass eine Lichttherapie der Schlaffragmentierung entgegenwirken und die Schlafdauer verlängern kann [23]. Falls wegen bestehender Augenleiden Bedenken hinsichtlich der Lichttherapie angebracht sind, sollte zuvor ein $\mathrm{Au}$ genarzt zurate gezogen werden.

\section{Fazit für die Praxis}

- Schlafstörungen bei Demenz können als Insomnie, Hypersomnie, Schlafapnoe, exzessive nächtliche motorische Aktivität, zirkadiane Schlaf-WachRhythmusstörungen und als Sun downing auftreten.

- Der erste Schritt der Diagnostik ist die ausführliche Schlafanamnese, die die Vorgeschichte und aktuelle Probleme mit Fokus auf Symptomatik und zugrunde liegender neurodegenerativer Erkrankung einbezieht. Der Einsatz des Pittsburgh Schlafqualitätsindex und der Epworth Sleepiness Scale sowie die Führung eines Schlaftagebuchs werden empfohlen. Mithilfe der Aktographie können zirkadiane Schlaf-Wach-Rhythmusstörungen dokumentiert werden.

- Die Therapie von Schlafstörungen bei Demenz sollte bei nichtpharmakologischen Interventionen ansetzen; diese müssen einer pharmakologischen Therapie vorausgehen und sollten diese zumindest begleiten.

- Wesentlicher Bestandteil der Therapie sind Aufklärung und Beratung, Vermittlung von Maßnahmen der Schlafhygiene, verschiedene Entspannungs- 
techniken und psychotherapeutische Interventionen.

- Die Verabreichung von (Psycho-)Pharmaka muss nach klarer Indikation, zeitlich begrenzt und unter klinischer Beobachtung erfolgen. Eine gute Orientierung bieten hier die Therapieempfehlungen der Schweizer Fachgesellschaften für BPSD, die Anfang 2014 publiziert wurden.

\section{Korrespondenzadresse}

\section{Prof. Dr. E. Savaskan}

Klinik für Alterspsychiatrie

Psychiatrische Universitätsklinik Zürich

Minervastr. 145, Postfach 16688032 Zürich

egemen.savaskan@puk.zh.ch

\section{Einhaltung ethischer Richtlinien}

Interessenkonflikt. E. Savaskan erklärt, dass kein Interessenkonflikt vorliegt.

Dieser Beitrag beinhaltet keine Studien an Menschen oder Tieren.

\section{Literatur}

1. Alford C, Verster J (2005) NICE review: not nice for patients! J Psychopharmacol 19(2):129-132

2. Ballard C, Howard R (2006) Neuroleptic drugs in dementia: benefits and harm. Nat Rev Neurosci 7:492-500

3. Beland SG, Preville M, Doubois MF et al (2010) Benzodiazepine use and quality of sleep in the community-dwelling elderly population. Agings Ment Health 14(7):843-850

4. Camargos EF, Pandolfi MB, Freita MP et al (2011) Trazodone for the treatment of sleep disorders in dementia: an open-label, observational and review study. Arq Neuropsiquiatr 69:44-49

5. Chokroverty S (2009) Sleep and neurodegenerative diseases. Semin Neurol 29:446-467

6. Dowling GA, Burr RL, Van Sommeren EJ et al (2008) Melatonin and bright light treatment for rest-activity disruption in institutionalized patients with Alzheimer's disease. J Am Geriatr Soc 56:239246

7. Fauth EB, Gibbons A (2014) Which behavioral and psychological symptoms of dementia are the most problematic? Variability by prevalence, intensity, distress ratings, and association with caregiver depressive symptoms. Int J Geriatr Psychiatry 29:263-271

8. Fick DM, Cooper JW, Wade WE et al (2003) Updating the Beers criteria for potentially inappropriate medication use in older adults. Arch Intern Med 163:2716-2724

9. Gehrman PR, Connor DJ, Martin JL et al (2009) Melatonin fails to improve sleep or agitation in double-blind randomized placebo-controlled trial of institutionalized patients with Alzheimer's disease. Am J Geriatr Psychiatry 17:166-169
10. Glass J, Lanctot KL, Herrmann N et al (2005) Sedative hypnotics in older people with insomnia: metaanalysis of risk and benefits. BMJ 331(7526):1169

11. Guillard J, Cheref S, Vacherontrystam MN, Martin JC (2002) Chloral hydrat: a hypnotic best forgotten? Encephale 28:200-204

12. Hatzinger M, Mathis J (2011) Insomnia. Schweiz Arch Neurol Psychiatr 162(8):310-317

13. Ju Y-ES, Lucey B, Holtzman DM (2014) Sleep and Alzheimer disease pathology-a bidirectional relationship. Nature Rev 10:115-119

14. McCleery J, Cohen DA, Sharpley AL (2014) Pharmacotherapies for sleep disturbances in Alzheimer's disease. Cochrane Database Syst Rev 3:CD009178

15. McCurry SM, Vitiello MV, Gibbons LE et al (2006) Factors associated with caregiver reports of sleep disturbances in persons with dementia. Am J Geriatr Psychiatry 14:112-120

16. Mizuno S, Kameda A, Inagaki T et al (2004) Effects of donepezil on Alzheimer's disease: the relationship between cognitive function and rapid eye movement sleep. Psychiatry Clin Neurosci 58:560 565

17. Moraes Wdos S, Poyares DR, Guilleminault C (2006) The effect of donepezil on sleep and REM sleep EEG in patients with Alzheimer disease: a doubleblind placebo-controlled study. Sleep 29:199-205

18. Mosimann UP, Müri RM (2011) Lewy-KörperchenDemenz - Ein Update. Schweiz Arch Neurol Psychiatr 162:102-107

19. Peter-Derex L, Yammine P, Bastuji H et al (2015) Sleep and Alzheimer's disease. Sleep Med Rev 19:29-38

20. Raji MA, Brady SR (2001) Mirtazapine for treatment of depression and comorbidities in Alzheimer disease. Ann Pharmacother 35:1024-1027

21. Roth HL (2012) Dementia and Sleep. Neurol Clin 30:1213-1248

22. Rothman SM, Mattson M (2012) Sleep disturbances in Alzheimer's and Parkinson's diseases. Neuromol Med 14:194-204

23. Salami O, Lyketsos C, Rao V (2011) Treatment of sleep disturbance in Alzheimer's dementia. Int J Geriatr Psychiatry 26:771-782

24. Savaskan E, Bopp-Kistler I, Buerge M et al (2014) Empfehlungen zur Diagnostik und Therapie der behavioralen und psychologischen Symptome der Demenz (BPSD). Praxis 103(3):135-148

25. S 3-Leitlinie Demenzen. Deutsche Gesellschaft für Psychiatrie, Psychotherapie und Nervenheilkunde (DGPPN) und Deutsche Gesellschaft für Neurologie (DGN) in Zusammenarbeit mit der Deutschen Alzheimer Gesellschaft e. V. - Selbsthilfe Demenz. Springer Berlin Heidelberg. 2010

26. Taibi DM, Landis CA, Petry H, Vitiello MV (2007) A systematic review of valerian as a sleep aid: safe but not effective. Sleep Med Rev 11:209-232 